\title{
Folic acid: Is self reported use of supplements accurate?
}

\author{
A Burton, S Wilson, A J Gillies
}

Low folate levels before conception and in early pregnancy are associated with an increased risk of neural tube defects (NTDs). ${ }^{1}$ Preconception folic acid supplements provide protection against NTDs. ${ }^{2}$ Self administered questionnaires are a simple and non-invasive method of obtaining estimates of preconception folic acid use, ${ }^{3}$ however, their accuracy is often dependent on the subject matter. Undesirable behaviours (for example, smoking, criminal offences) are generally under-reported while desirable behaviours (for example, exercise, condom use) are often over-reported. Taking folic acid supplements to prevent NTDs is a desirable outcome and therefore over-reporting may be apparent. Haematological measures for both current and cumulative use of folic acid supplement provide accurate indicators of a woman's folate status and therefore provide good tools against which to validate self reporting. If self reporting is a valid indicator of folic acid supplementation then this may be quicker, cheaper and more acceptable to patients than collecting blood samples. This study aimed to assess the validity of self reported supplement use by comparison with haematological measurements.

\section{Methods}

A questionnaire was self administered by consenting women, attending three hospital-based antenatal clinics within the West Midlands region of the United Kingdom, who also provided a blood sample. ${ }^{4}$ Three questions assessed the use of folic acid supplements: (1) Have you taken folic acid at any point before or during this pregnancy? (2) Did you take folic acid before you got pregnant? (3) Are you taking folic acid at this moment? The questionnaires were administered during 1996-97 and also collected demographic and dietary information. Serum and red cell folate (RCF) measurements were made at a single laboratory

Accepted for publication 10 July 2001

Table 1 Relation between reported use of folic acid supplements and blood folate levels

\begin{tabular}{|c|c|c|c|c|c|c|}
\hline \multicolumn{3}{|c|}{ Use of folic acid } & \multirow[b]{2}{*}{$(N)$} & \multicolumn{3}{|c|}{ Folate levels } \\
\hline \multirow[t]{2}{*}{ Ever taken } & $\begin{array}{l}\text { Took before } \\
\text { conception }\end{array}$ & Taking now & & Median & $I Q R^{\star}$ & Range \\
\hline & & & & \multicolumn{3}{|c|}{ Serum Folate levels $(\mathrm{nmol} / \mathrm{l})$} \\
\hline No & No & No & $(379)$ & 13 & $9-20$ & $2-73$ \\
\hline Yes & No & No & $(271)$ & 18 & $12-27$ & $4-126$ \\
\hline Yes & Yes & No & $(128)$ & 24 & $17-34$ & $4-72$ \\
\hline Yes & No & Yes & $(404)$ & 31 & $21-47$ & $3-344$ \\
\hline \multirow[t]{2}{*}{ Yes } & Yes & Yes & $(247)$ & 40 & $29-52$ & $10-442$ \\
\hline & & & & \multicolumn{3}{|c|}{ Red cell folate levels (nmol/l) } \\
\hline No & No & No & $(370)$ & 358 & $270-471$ & $7-1206$ \\
\hline Yes & No & No & (269) & 462 & $367-596$ & $0-3032$ \\
\hline Yes & No & Yes & (393) & 492 & $374-664$ & $23-2411$ \\
\hline Yes & Yes & No & $(122)$ & 658 & $444-816$ & $150-1826$ \\
\hline Yes & Yes & Yes & $(241)$ & 693 & $553-877$ & $45-1876$ \\
\hline
\end{tabular}

$\star \mathrm{IQR}=$ interquartile range. with a standard test (BIO-RAD Quantaphase II $\mathrm{B} 12 /$ Folate radioassay) to minimise measurement error. The laboratory was blinded to patients' use of folic acid supplements to avoid any bias. Data were reported by the laboratory in conventional units $(\mu \mathrm{g} / \mathrm{l})$ and converted to SI units ( $\mathrm{nmol} / \mathrm{l}$ ) by multiplying by a conversion factor of 2.266 .

The combinations of yes/no responses to the questions on folic acid use were classified into five ordered groups according to short-term and long term use separately (table 1 ). RCF is a measure of cumulative intake of folic acid and associated with long term use whereas serum folate, a measure of short-term intake, is associated with recent use of folic acid. Summary statistics for both the serum and RCF measurements were calculated for the five groups. The Wilcoxon type test for trend was used to assess folate levels across the ordered groups.

Over-reporting was defined as self reported pre-conception or recent supplementation use with an associated low RCF or folate level $(\mathrm{RCF}<453 \mathrm{nmol} / 1$ or serum folate $<14$ nmol/1) respectively.

\section{Results}

Altogether 1485 (94\%) of 1575 women attending the clinics provided consent. The median age was 28 years (range 14-47), the median gestational age was 13 weeks and 534 $(36 \%)$ were in their first pregnancy. The majority of the women $(78 \%)$ were white, with $15 \%$ Asian, $5 \%$ Afro-Caribbean and $2 \%$ from other ethnic backgrounds or not specified. The median Townsend score (derived from postcodes) was 1.6 (interquartile range -1.6 to 4.6). Altogether 388 (26\%) women reported taking folic acid preconception but only 194 $(13 \%)$ reported taking folic acid supplements as recommended by the Department of Health (that is, preconception and 12 weeks postconception). ${ }^{5}$

The median serum folate measurement was $24 \mathrm{nmol} / \mathrm{l}$ (interquartile range (IQR): 13-38 $\mathrm{nmol} / \mathrm{l})$ and median $\mathrm{RCF}$ was $482 \mathrm{nmol} / \mathrm{l}$ (IQR: $349-678 \mathrm{nmol} / \mathrm{l}$ ). There was a significant linear trend in serum folate levels as current use of supplements increased $(z=-21.97$, $\mathrm{p}<0.0001$, table 1). RCF levels also increased with increasing reported cumulative use of supplements $(z=-17.90, p<0.0001)$. The ranges of serum and RCF measurements between the folic acid groups were not distinct and there was wide variation of values within groups (table 1).

Over-reporting of supplement use was apparent for a maximum of 109 (7\%) women. 


\section{Discussion}

Median serum and RCF concentrations for all participants were $24 \mathrm{nmol} / 1$ and $482 \mathrm{nmol} / \mathrm{l}$ respectively. These levels are lower than those reported by a recent USA population based series. ${ }^{6}$ Higher reported levels in the USA may be attributable to a different sampling frame (we only surveyed pregnant women) or the implementation of food fortification and educational initiatives aiming to increase folate consumption.

Previous studies, based on comparatively small samples of patients, have demonstrated an association between the dose of folic acid supplementation and RCF levels. ${ }^{7}$ However, we have not identified any previous publications reporting the relation between self reported folic acid supplementation use and either serum or RCF levels. The significant linear trend between self reported folic acid supplementation and folate status as measured by both serum folate and RCF suggests that as women report taking more folic acid, and for longer, their serum and RCF levels increase correspondingly. It also suggests that self reporting provides a valid method of assessing folic acid supplementation and is suitable for assessing the effectiveness of public health intervention strategies within the population of pregnant women. Non-invasive methods for monitoring the use of folic acid supplementation have advantages in terms of time, cost and compliance. It is concluded that future population based assessments of folic acid use can be based on self reports of supplementation.

Our thanks to the consultants, staff and women at the three antenatal clinics at Birmingham Women's Hospital NHS trust, Good Hope Hospital NHS trust, Sutton Coldfield and Birmingham Heartlands Hospital NHS trust. Also thanks to Sandy McLeod for liaising with the hospitals and data collection, Mr John George and staff at the Department of Haematology, University Hospital Birmingham NHS trust and Professor Richard Lilford.

Funding: NHSE (West Midlands).

Conflicts of interest: none.

1 Christensen B, Rosenblatt DS. Effects of folate deficiency on embryonic development. Baillieres Clin Haematol on embryonic

2 MRC Vitamin Study Research Group. Prevention of neural tube defects: results of the Medical Research Council Vitamin Study. Lancet 1991;338:131-7.

3 Clark NAC, Fisk NM. Minimal compliance with DoH recommendation for routine folate prophylaxis to prevent neural tube defects. Br F Obstet Gynaecol 1994;101:709-10.

4 Gilles A, McCleod S, Wilson S, et al. Periconception folic acid supplementation: Women's knowledge and Haematological values in pregnancy 18 months into the HEA Folic Acid Campaign. Birmingham: R\&D Directorate NHSE (West Midlands) report, 1999.

5 Department of Health. Folic acid and the prevention of neural tube defects. Report from an expert advisory group. London, HMSO, 1992.

6 Centre for Disease Control. Folate status in women of childbearing age-United States, 1999. MMWR 2000;49: 962-5.

7 Daly S, Mills JL, Molloy AM, et al. Minimum effective dose of folic acid for food fortification to prevent neural tube of folic acid for food fortification 